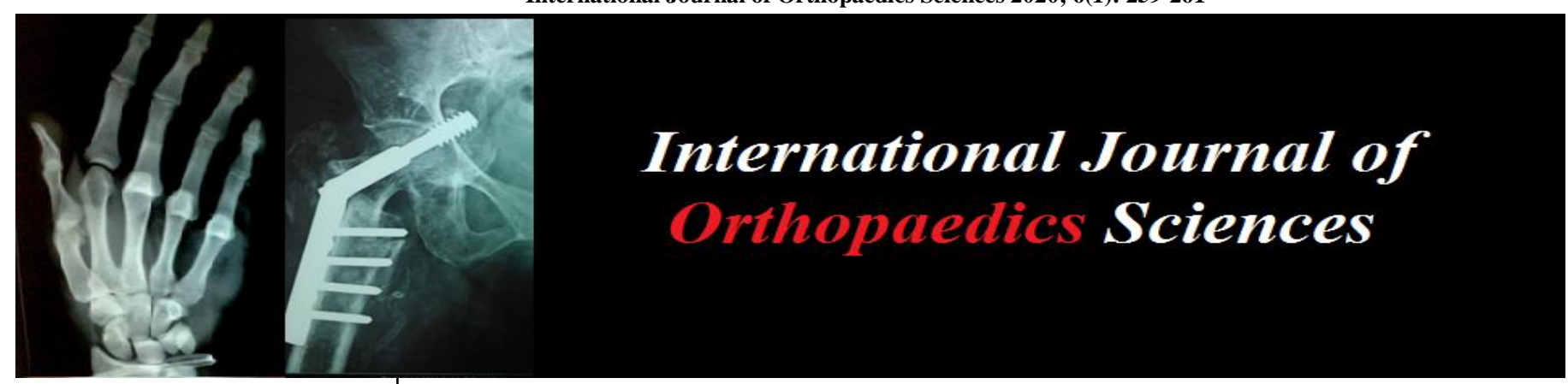

E-ISSN: 2395-1958

P-ISSN: 2706-6630

IJOS 2020; 6(1): 259-261

(C) 2020 IJOS

www.orthopaper.com

Received: 10-11-2019

Accepted: 12-12-2019

Dr. Sanjeevkumar Munoli Assistant Professor, Department of General Surgery, Mahavir Institute of Medical Sciences, Vikarabad, Telangana, India
Corresponding Author: Dr. Sanjeevkumar Munoli Assistant Professor, Department of General Surgery, Mahavir Institute of Medical Sciences, Vikarabad, Telangana, India

\section{A study of lumbar spine surgeries under spinal anaesthesia}

\section{Dr. Sanjeevkumar Munoli}

DOI: https://doi.org/10.22271/ortho.2020.v6.i1e.1876

\section{Abstract}

Introduction: Spinal anaesthesia was used in selected patients undergoing lumbar spine surgery in Maheshwara Medical College, Chitkul, Hyderabad. The technique will be described in detail and the results will be reported.

Materials and Methods: This study reports on patients admitted and operated upon from 1st Aug 2016 till 31st July 2017. We collected the data of all the patients operated under regional anaesthesia (Spinal Anaesthesia) and followed them up at 6 months, 12 months and 24 months post-operatively. The demographics, anaesthesia details, operation details, complications if any and short-term, medium-term (two-year) post-op outcome during follow-up visits were noted down for the present study.

Results: Thirty six out of 52 patients had simple lumbar spinal operations performed under spinal anaesthesia in the period under review. There were 25 males and 11 females with age range 24-65 years. 26 out of the 36 patients had one level lumbar spine decompression, while the remaining 10 had twolevel spinal decompression performed. None of the patients required blood transfusion, and none of the patients had anaesthesia-related or surgery-related complications intra-operatively or Post-operatively (except one patient who had 2 episodes of vomiting). There were no complications such as dural tears, nerve injuries related to the procedures. The post-operative analgesia was maintained with intravenous infusion of Diclofenac (aqueous preparation) for 8-12hours. On review after 6months, 12months and 24 months post-operatively, the patients and the surgeon were satisfied with spinal anaesthesia in all cases.

Conclusion: Lumbar spine surgeries can be performed safely under regional (Spinal) anaesthesia. For patients undergoing lumbar spine decompressive surgery, regional (Spinal) anaesthesia is an effective technique with potential advantages. In our limited experience, the operations were well tolerated by patients with good recovery and minimal complications. The short-term and medium-term outcomes were also satisfactory.

Keywords: spinal anaesthesia; lumbar decompressive surgery

\section{Introduction}

Spine procedures can be performed either under general or regional anaesthesia. However, general anaesthesia is the anaesthetic technique used frequently. This is the usual technique for prolonged surgeries in the prone position. It is generally preferred for patient's comfort/tolerance and to prevent airway compromise. While some think all lumbar spine surgeries should be done under general anaesthesia to achieve better outcomes, studies have shown that short-duration procedures like simple lumbar-discectomy and laminectomy can be successfully done under spinal anaesthesia with good outcomes and patient's tolerance/satisfaction ${ }^{[1]}$. Lumbar spine surgery can be performed with safety under regional anaesthesia ${ }^{[2]}$. Regional anaesthesia is used for simple procedures (which can be completed in short-duration) in carefully selected patients. For patients undergoing decompressive lumbar spine surgery, epidural anesthesia is an effective, well tolerated technique with several potential advantages. Mclain et al. in a case controlled study looked at 400 patients who underwent either spinal anaesthesia or general anaesthesia for lumbar decompression surgeries. The results showed that spinal anaesthesia was as effective as general anaesthesia, and in addition spinal anaesthesia caused reduced anaesthesia recovery duration, reduction in nausea and vomiting and post-op analgesia needs ${ }^{[3]}$. Tetzlaff et al. also retrospectively looked into the outcomes of elective lumbar spine surgical procedures done under 
spinal anaesthesia or general anaesthesia. They concluded that spinal anaesthesia can be considered an effective alternative to general anaesthesia for lumbar spine surgery. They noticed reduced blood loss in the patients who had spinal anaesthesia and reduced post operative analgesic use ${ }^{[4]}$.

In our study, patients for Lumbar spine operations were chosen for spinal anaesthesia only if: decompression of spine not to exceed 2 levels and the anticipated surgery duration not more than 2 hours. Spinal anaesthesia was performed in carefully selected patients undergoing lumbar spine surgery in Maheshwara Medical College and hospital. We present our experience about lumbar spine surgeries done under regional/spinal anaesthesia.

\section{Materials and Methods}

We conducted a prospective study of our patients admitted for spine surgery during the study period, from 1st Aug' 2016 to 31st July'2017. A database was created and the patient demographics, anaesthesia technique, operation-details were entered in respective case files. All the 36 patients were ASA 1 or 2 patients scheduled for elective discectomy or laminectomy and nerve root decompression, who did not have any contraindication to spinal anaesthesia. The patients were all operated upon by the same team of neurosurgeon, anaesthetist and perioperative nurse. Routine preoperative assessment was done to ascertain patients fitness and to rule out coagulopathy, hypovolaemia, infection at injection site, history of seizure and raised intra cranial pressure. An informed consent was obtained after careful explanation of the anaesthetic technique and what to expect in the perioperative period was communicated to the patients. Fasting guidelines (4-6hours prior to operation) were given. After shifting to operation theatre routine monitoring of noninvasive blood pressure, oxygen saturation, pulse, temperature and electrocardiogram were done, and continued throughout the surgery and later in the recovery room. A wide bore cannula of $16 \mathrm{G}$ was inserted, secured and patients preloaded with normal saline $7 \mathrm{ml} / \mathrm{kg}$ over 15 minutes. The patients were placed in the sitting position/right-lateral/left-lateral depending on patient's comfort. The back was cleaned and draping performed in an aseptic technique. The space to be used for spinal anaesthesia, usually L3/L4 (occasionally L2L3) was infiltrated with $3-4 \mathrm{ml}$ of $2 \%$ Lidocaine. Then, a $25 \mathrm{~g}$ spinal needle was introduced into the spine to the subarachnoid space until clear cerebrospinal fluid is observed. $3 \mathrm{ml}$ of heavy Bupivacaine was then injected into the subarachnoid space to produce the spinal anaesthesia. Patients were made to lie down supine for 10-15 minutes. Meanwhile, an appropriate size catheter was placed aseptically into the urinary bladder; routine prophylactic antibiotic was also given. We regularly use $1 \mathrm{gm}$ Ceftriaxone given intravenously. After establishing level of spinal block at T6-T8, patients were then turned prone on bolsters to free the stomach and chest. Soft pillows were also used to support the head, limbs and to protect the pressure points. All the surgeries were performed by the same team of surgeons. Throughout the surgery, blood pressure was monitored and hypotension (systolic blood pressure less than $90 \mathrm{mmHg}$ ) was treated with injections of Ephedrine/Mephenteramine intravenously. Blood loss during procedure was monitored and recorded in the anaesthetic chart. At the end of surgery, patients were turned back into the supine position, propped up to about 30degrees, taken out of theatre and monitored in the recovery room. Recovery time was documented as time from arrival at the recovery room to shifting to regular room/ward. In the recovery room, the following were assessed-Pain, nausea/vomiting, vital signs, regression of sensory block to at least 2 segments below initial level. Patients' acceptance/tolerance and surgeon's satisfaction was also assessed in simple Yes or No question format after the operation. The medium-term outcome was assessed at 6 months, 12 months and 24 months. We confirmed that patients continued to have good outcome following the operation, with simple Satisfied or not satisfied question format.

\section{Results}

Data of the patients admitted and operated upon from 1st Aug 2016 till 31st July 2017 at our institute suggested that 36 out of 52 patients had simple lumbar spinal operations performed under spinal anaesthesia. There were 25 males and 11 females with age range $24-65$ years. 26 out of the 36 patients had one level lumbar spine decompression, while the remaining 10 had two-level spinal decompression. None of the patients had anaesthetic or surgical complications perioperatively. The average duration of the procedures was 70 minutes. Blood loss was estimated between $50-100 \mathrm{ml}$ and no one required a blood transfusion. There were no complications such as dural tears, nerve injuries related to the procedures and patients were able to eat and drink 3-4 hours after the operation. One patient vomited twice in the recovery room. The post operative analgesia was maintained for over 8-12hours post operatively. On review immediate Post-operatively, at 6months, 12 months \& 24months Post-operatively, the patients and the surgeon were satisfied with spinal anaesthesia in all cases.

\section{Discussion}

Our limited experience suggested that patients found it difficult to believe that spine surgeries can be successfully performed under Spinal anaesthesia. Spine surgeries have been successfully done under general anaesthesia, spinal and epidural anaesthesia. Many studies have been done to establish the feasibility and safety of regional anaesthesia in lumbar spine surgery ${ }^{[3,4,5]}$. It had long been used in obstetrics with good safety profile ${ }^{[6]}$. Application of regional anesthesia is also widely preferred for lower-extremity surgery, but general anesthesia is used almost exclusively in spine surgery, despite evidence that spinal anesthesia is as safe and may offer some advantages ${ }^{[4,5]}$. We discovered that having the operation performed under regional anaesthesia increased the take up rate for surgery in our Institute. For those we have offered the option of spinal anaesthesia for short-duration spinal procedures, it came as a relief that they could be awake and have their procedure done safely in this way. There needs to be careful patient selection and adequate informed consent. The patient need to be co-operative and tolerant of mild discomfort related to the prone position. The operation should also not last much more than 2 hours. The operations were performed under spinal anaesthesia though it could also be performed under epidural anaesthesia [5, 7]. In fact, other authors have reported successful rates of motor and sensory blockade (over 90\%) even in patients who had undergone previous spine surgery ${ }^{[8]}$. Causes for failure of extradural anaesthesia in patients who had previously undergone lumbar spine surgery included technical difficulty in three patients and inadequate spread in two patients. The reported frequency of serious complications is low and they were usually due to the spread of anaesthesia, leading to circulatory and respiratory insufficiency ${ }^{[8]}$. It is therefore essential to be able 
to treat such complications rapidly ${ }^{[7,9]}$. In our experience, the operation was well tolerated by patients with good recovery and minimal complications. We had only one patient out of the 36 cases operated upon, who suffered 2 episodes of vomiting following the operation. We have not adopted spinal anaesthesia as a choice of anaesthesia for patients, who in addition to the decompression required pedicle screw fixation. This is because pedicle screw fixation takes a longer time, and patients may not be able to stay awake in the prone position, and be comfortable for procedures longer than 2 hours. One important point is that patients need to be warned about the risk of conversion to general anaesthesia. As reported by Hassi et al. the possible risk of failure obliges to inform patients preoperatively that conversion to general anaesthesia cannot be excluded ${ }^{[2]}$.

Spinal anaesthesia is advantageous for less risk of some complications compared with general anaesthesia and enables communication between the surgeon and the patient. Another problem with regional anaesthesia is the risk of hypotension. Ephedrine/Mephenteramine is used frequently to manage the problem. Laakso et al. concluded that there was a tendency to more frequent episodes of haemodynamic deterioration in the knee-chest position than the horizontal position ${ }^{[10]}$. All our patients were operated lying in the horizontal position. Some other reported complications are the risk of hyperacute spinal subdural haematoma secondary to lumbar spinal anaesthesia, identified with MRI as reported [9] and lumbar spondylodiscitis after epidural anaesthesia at a distant site ${ }^{[11]}$. In our study we have noticed that both surgeon and patients had a high level of satisfaction after the procedure.

\section{Conclusion}

Spinal anaesthesia is a reliable and satisfactory alternative form of anaesthesia for simple lumbar spine procedure. Spinal anaesthesia for short spinal procedures is an option that should be employed in suitable cases. The team must be aware of possible complications such as hypotension, failure of the blockade and more potentially serious complications as paraplegia Ouro-Bang'na Maman et al. ${ }^{[12]}$. Overall, in our limited experience, the patients and surgeons were satisfied at the end of the procedures.

\section{References}

1. Smrcka M, Baudysova O, Juran V, Vidlak M, Gal R et al. Lumbar disc surgery in regional anaesthesia-40 years of experience. Acta Neurochir (Wien). 2001; 143:377381.

2. Hassi N, Badaoui R, Cagny-Bellet A, Sifeddine S, Ossart M. Spinal anesthesia for disk herniation and lumbar laminectomy. Apropos of 77 cases. Cah Anesthesiol. 1955; 43:21-25.

3. McLain RF, Kalfas I, Bell GR, Tetzlaff JE, Yoon HJ et al. Comparison of spinal and general anesthesia in lumbar laminectomy surgery: a case-controlled analysis of 400 patients. J Neurosurg Spine. 2005; 2:17-22.

4. Tetzlaff JE, Dilger JA, Kodsy M, Al-Bataineh J, Yoon HJ et al. Spinal anesthesia for elective lumbar spine surgery. J Clin Anesth. 1998; 10:666-669.

5. Downing JW, Houlton PC, Brock-Utne JG, Mankowitz E. Lumbar epidural anaesthesia. S Afr Med J. 1979; 56:844-847.

6. Crawford JS. Experiences with lumbar extradural analgesia for caesarean section. Br J Anaesth. 1980; 52:821-825.

7. Jorgensen H. Lumbar epidural anaesthesia with bupivacaine $0.75 \%$. A clinical evaluation of 371 cases. Reg Anaesth. 1982; 5:30-33, 10.

8. Sharrock NE, Urquhart B, Mineo R. Extradural anaesthesia in patients with previous lumbar spine surgery. Br J Anaesth. 1990; 65:237-239.

9. Pedraza Gutierrez S, Coll Masfarre S, Castano Duque $\mathrm{CH}$, Suescun M, Rovira Canellas A. Hyperacute spinal subdural haematoma as a complication of lumbar spinal anaesthesia: MRI. Neuroradiology. 1999; 41:910-914, 11.

10. Laakso E, Pitkanen M, Kytta J, Rosenberg PH. Kneechest vs horizontal side position during induction of spinal anaesthesia in patients undergoing lumbar disc surgery. Br J Anaesth. 1997; 79:609-611.

11. Halkic N, Blanc C, Corthesy ME, Corpataux JM. Lumbar spondylodiscitis after epidural anaesthesia at a distant site. Anaesthesia. 2001; 56:602-603, 14.

12. Ouro-Bang'na, Maman AF, Tomta K, Songne B, Moumouni I, Abalo A. Paraplegia after spinal anaesthesia in a patient presenting a degenerative lumbar spinal disease]. Ann Fr. Anesth Reanim. 2007; 26:465-466. 